\title{
Gemeinwohlinteressen und Grundfreiheiten: zum \\ Gestaltungsspielraum der Mitgliedstaaten bei der Rechtfertigung des Eingriffs in Grundfreiheiten
}

\author{
Astrid Epiney
}

\begin{abstract}
Dieser Beitrag wurde erstmals wie folgt veröffentlicht:
Astrid Epiney, Gemeinwohlinteressen und Grundfreiheiten: Zum Gestaltungsspielraum der Mitgliedstaaten bei der Rechtfertigung des Eingriffs in Grundfreiheiten, FS Peter-Christian Müller-Graff, Baden-Baden 2015, 467-474. Es ist möglich, dass diese publizierte Version - die allein zitierfähig ist - im Verhältnis zu diesem Manuskript geringfügige Modifikationen enthält.
\end{abstract}

\section{Einleitung}

Die durch den AEU-Vertrag eröffnete Möglichkeit, einen Eingriff in die Grundfreiheiten zu rechtfertigen, ist vor dem Hintergrund zu sehen, dass die Grundfreiheiten - die das Herzstück des Binnenmarkts darstellen (vgl. auch Art. 26 Abs. 2 AEUV) und denen für das EU-Recht insgesamt eine zentrale Bedeutung zukommt - nicht dazu führen sollen, dass es den Mitgliedstaaten verunmöglicht wird, berechtigte Anliegen des Allgemeinwohls (m.a.W. Gemeinwohlinteressen) zu verfolgen. Trotz einer weit verzweigten und quantitativ wie qualitativ reichhaltigen Rechtsprechung des Gerichtshofs zum Thema ${ }^{1}$ dürfte aber nach wie vor nicht abschließend geklärt sein, wie weit genau der Gestaltungsspielraum der Mitgliedstaaten in diesem Zusammenhang geht, dies obwohl dieser Gesichtspunkt für Verfolgung von Gemeinwohlinteressen durch die Mitgliedstaaten von zentraler Bedeutung ist.

Vor diesem Hintergrund sollen im Folgenden, auf der Grundlage einer Skizzierung der dogmatischen Struktur und des Hintergrunds der Möglichkeit der Rechtfertigung von Eingriffen in die Grundfreiheiten (II.), die Gestaltungsspielräume der Mitgliedstaaten präzisiert (III.) und damit einen Beitrag zur Dogmatik der Grundfreiheiten (ein Rechtsgebiet, mit dem sich auch der Jubilar verschiedentlich und vertieft befasst hat $)^{2}$ geleistet werden, woran die sich ein kurzes Schlusswort anschliesst (IV.). ${ }^{3}$

S. den Überblick bei Astrid Epiney, in: Bieber/Epiney/Haag, Die Europäische Union, 2013, § 10, Rn. 15 ff. S. jüngst Peter-Christian Müller-Graff, Die horizontale Direktwirkung der Grundfreiheiten, EuR 2014, 3 ff.

Dabei wird nachfolgend auf Literaturnachweise verzichtet. Zu den Grundfreiheiten die Kommentarliteratur sowie Dirk Ehlers (Hrsg.), Europäische Grundrechte und Grundfreiheiten, 3. Aufl., 2009 (4. Aufl. im Erscheinen); Walter Frenz, Handbuch Europarecht. Europäische Grundfreiheiten, 2. Aufl., 2012. 


\section{Die Rechtfertigung von Eingriffen in die Grundfreiheiten: Hintergrund und Struktur}

Die Möglichkeit der Rechtfertigung von Eingriffen in die Grundfreiheiten ist in erster Linie vor dem Hintergrund zu sehen, dass die Grundfreiheiten beschränkende Maßnahmen zur Verwirklichung öffentlicher Interessen notwendig sein können und dass der Schutz der betreffenden Rechtsgüter auf Unionsebene nicht oder nicht erschöpfend geregelt ist, so dass ein legitimes Interesse daran bestehen kann, zum Schutz von Gemeinwohlinteressen die Grundfreiheiten einzuschränken. Im Übrigen wird die Verfolgung der in diesem Zusammenhang relevanten Schutzinteressen in aller Regel im Bereich der geteilten Zuständigkeiten (Art. 4 AEUV) liegen, so dass die Mitgliedstaaten solange und soweit zur Regelung der betreffenden Bereiche zuständig sind, wie die Union ihre Kompetenzen nicht wahrgenommen hat. Dabei haben sie selbstverständlich die Vorgaben des Unionsrechts zu beachten, zu denen auch die Grundfreiheiten gehören; jedoch ist gerade bei der Frage nach den Gestaltungsspielräumen der Mitgliedstaaten auch diese grundsätzliche Regelungskompetenz der Mitgliedstaaten zu beachten. ${ }^{4}$

Vor diesem Hintergrund erklären sich auch die maßgeblich durch die Rechtsprechung entwickelten Voraussetzungen der Rechtfertigung eines Eingriffs in die Grundfreiheiten (die in den Grundsätzen wohl parallel ausgestaltet sind): ${ }^{5}$

- $\quad$ Erstens muss der Eingriff durch solche Gemeinwohlinteressen begründet sein, die durch das Unionsrecht anerkannt sind. Dabei sind die geschriebenen (Art. 36, 45 Abs. 2, 52 AEUV) von den ungeschriebenen Rechtfertigungsgründen (,zwingende Erfordernisse des Allgemeinwohls" ${ }^{6}{ }^{6}$ zu unterscheiden. Unter letztere können letztlich alle öffentlichen Interessen subsumiert werden, mit Ausnahme der sog. wirtschaftlichen Gründe (also solche, die auf die Wirtschaftslenkung oder die Verfolgung sonstiger wirtschaftspolitischer Anliegen abzielen): Diese können keinesfalls einen Eingriff in die Grundfreiheiten rechtfertigen, ${ }^{7}$ da dies Idee und Zielsetzung der Grundfreiheiten selbst zuwiderliefe.

S.u. III.

Hierzu die Nachweise in Fn. 1, 3.

Grundlegend für den freien Warenverkehr EuGH, Rs. 120/78 (Rewe), Slg. 1979, 649, Rn. 8, 14 (Cassis de Dijon).

$7 \quad$ S. z.B. EuGH, Rs. C-324/93 (Evans), Slg. 1995 I-563, Rn. 36; EuGH, Rs. C-398/95 (Syndesmos ton Elladi Touristikon), Slg. 1997, I-3091, Rn. 22 f.; EuGH, Rs. C-220/12 (Thiele Meneses), Urt. v. 24.10.2013. 
- Zweitens muss den Anforderungen der Verhältnismäßigkeit Genüge getan sein, ${ }^{8}$ kann doch nur unter dieser Voraussetzung davon ausgegangen werden, dass tatsächlich der Verwirklichung der verfolgten öffentlichen Interessen gedient ist.

\section{Zur Reichweite des Gestaltungsspielraums der Mitgliedstaaten}

\section{1. „Mitgliedstaatliche“ Gemeinwohlinteressen}

Wenn die Möglichkeit der Rechtfertigung von Eingriffen in die Grundfreiheiten den Mitgliedstaaten die Verfolgung öffentlicher Interessen erlauben soll und wenn den Mitgliedstaaten insoweit eine geteilte Kompetenz zukommt (sowie die Union ihre Kompetenzen nicht oder nicht erschöpfend wahrgenommen hat), muss die Entscheidung, ob ein bestimmtes Interesse als öffentliches Interesse anzusehen und zu verfolgen ist, durch die Mitgliedstaaten getroffen werden, so dass es hier letztlich um mitgliedstaatliche Gemeinwohlinteressen geht. Zu beachten sind allerdings die unionsrechtlichen Schranken, die jedoch nur sog. wirtschaftliche Gründe ausschließen, während alle sonstigen öffentlichen Interessen vom Unionsrecht grundsätzlich anerkannt sind.

Bei der Frage, ob es sich um „zulässige“ Allgemeinwohlinteressen handelt, geht es also nicht darum, ob diese (auch) auf Unionsebene anerkannt sind, sondern ausreichend ist ihre Verfolgung auf nationaler Ebene, immer unter der Voraussetzung, dass es sich nicht um wirtschaftliche Interessen handelt. Das Unionsrecht steht den Mitgliedstaaten hier also einen sehr weitgehenden Gestaltungsspielraum zu, was jedoch auf der Grundlage des skizzierten primärrechtlichen Hintergrundes zwingend ist, da dieser - über die Anerkennung der „Zwingenden Erfordernisse des Allgemeinwohls“ - den Mitgliedstaaten die Verfolgung aller Gemeinwohlinteressen erlaubt.

Auch die Rechtsprechung dürfte in diese Richtung gehen, auch wenn sie - soweit ersichtlich diesen Zusammenhang bislang noch nicht klar eindeutig formuliert hat. Zunächst prüft der EuGH regelmäßig lediglich, ob ein bestimmtes Interesse eventuell als wirtschaftlicher Grund anzusehen ist, ohne jedoch darüber hinaus seine Anerkennung auf unionsrechtlicher Ebene als entscheidend anzusehen (wenn auch mitunter hierauf hingewiesen wird). ${ }^{9}$ Sodann hat zahlreiche öffentliche Interessen als zwingende Erfordernisse des Allgemeinwohls (die er

Vgl. die Nachweise in Fn. 1, 3.

S. z.B. EuGH, Rs. C-379/98 (Preussen Elektra), Slg. 2001, I-2099, Rn. 73 ff.; EuGH, Rs. C-112/00 (Schmidberger), Slg. 2003, I-5659, Rn. 74. 
übrigens immer in nicht abschließender Form zitiert) anerkannt, ${ }^{10}$ darunter auch solche, die auf EU-Ebene wohl nicht als öffentliche Interessen anzusehen sind bzw. bei denen dies zumindest sehr fraglich wäre. So stellte der Gerichtshof z.B. bei der Frage, ob der Schutz der Menschenwürde - so wie er im deutschen Grundgesetz ausgestaltet ist - einen Eingriff in die Grundfreiheiten zu rechtfertigen vermag, entscheidend darauf ab, dass dieses Anliegen (das unter den Begriff der öffentlichen Ordnung subsumiert wurde) mit dem Unionsrecht „vereinbar“ sei, ohne dass es insoweit darauf ankomme, dass der Schutz der Menschenwürde in Deutschland eine besondere Stellung zukommt. ${ }^{11}$ In seiner Rechtsprechung zu Transferregeln im Profisport lässt der EuGH die Suche nach Talenten sowie die Ausbildung junger Sportler als zwingende Interessen des Allgemeinwohls gelten, dies obwohl es schwierig sein dürfte, diese Zielsetzungen als solche des Unionsrechts zu definieren; der Gerichtshof stellt denn auch auf ihre „Anerkennung“ durch das Unionsrecht ab. ${ }^{12}$

Dieser hier vertretene dogmatische Ansatz kann durchaus konkrete entscheidungserhebliche Implikationen zeitigen: So werden die verfolgten Schutzinteressen zwar in aller Regel auch auf Unionsebene als solche verfolgt (z.B. Umweltschutz, Verbraucherschutz); jedoch sind andere Konstellationen durchaus vorstellbar und auch - wie gezeigt - mitunter praktisch relevant, so z.B. soweit die tatbestandliche Reichweite grundrechtlicher Gewährleistungen auf mitgliedstaatlicher Ebene anders bzw. weiter ausgestaltet ist als auf EU-Ebene. Es wäre vor diesem Hintergrund $\mathrm{zu}$ wünschen, dass die Rechtsprechung diesen Aspekt des den Mitgliedstaaten zustehenden Gestaltungsspielraums noch deutlicher herausarbeitet; der in den Urteilen häufig zu findende Hinweis auf die (auch) unionsrechtliche Anerkennung des entsprechenden verfolgten Interesses ist nämlich mitunter missverständlich und trägt - da es hierauf eben gerade nicht bzw. nur unter der Rubrik der unionsrechtlichen „Akzeptanz“ ankommt - den hier formulierten Zusammenhängen nur bedingt Rechnung.

\section{Schutzkonzeption und Schutzniveau}

Wenn es bei den verfolgten öffentlichen Interessen um mitgliedstaatliche Interessen geht und wenn diesen - wie skizziert - schon aufgrund der Kompetenzverteilung zwischen Union und Mitgliedstaaten die Kompetenz zukommt, die entsprechende Schutzpolitik zu verfolgen, so

Vgl. die Nachweise in Fn. 1, 3.

EuGH, Rs. C-36/02 (Omega), Slg. 2004, I-9609, Rn. 33 f.

EuGH, Rs. C-415/93 (Bosman), Slg. 1995, I-4921, Rn. 105 ff.; s. auch EuGH, Rs. C-325/08 (Olympique Lyonnais), Slg. 2010, I-2177. 
muss konsequenterweise auch die Entscheidung darüber, welche Schutzkonzeption und welches Schutzniveau zugrunde gelegt wird, den Mitgliedstaaten obliegen. Es ist nicht ersichtlich, warum die Union (bzw. der Gerichtshof) ihre Konzeption an die Stelle derjenigen der Mitgliedstaaten setzen können soll, geht es hier doch nicht um die Definition unionsrechtlicher Begriffe, sondern um politische Akzente. Dieser Aspekt ist insbesondere deshalb von großer Bedeutung, weil Schutzkonzeption und -niveau für den Referenzpunkt der Verhältnismäßigkeitsprüfung ausschlaggebend sind, wird dadurch doch das maßgebliche Ziel definiert, an dem die Zulässigkeit der Mittel zu messen ist.

Die Rechtsprechung des EuGH trägt diesem Grundsatz nicht immer genügend Rechnung: Zwar betont der Gerichtshof regelmäßig (insbesondere im Zusammenhang mit dem Gesundheitsschutz, aber auch etwa im Bereich der Regulierung von Lotterien), es sei Sache der Mitgliedstaaten, das Schutzniveau festzulegen, so dass insbesondere der Umstand, dass man in anderen Mitgliedstaates ein niedrigeres Schutzniveau kenne, als solcher nichts an der Zulässigkeit der Maßnahme zu ändern vermöge. ${ }^{13}$ In anderen Bereichen jedoch greift der Gerichtshof auch im Zusammenhang mit der Frage nach der Rechtfertigung mitgliedstaatlicher Maßnahmen auf unionsrechtliche Konzepte zurück, dies obwohl der betreffende Mitgliedstaat eine andere Konzeption zugrundelegen möchte. Relevant ist dies in erster Linie im Bereich des Verbraucherschutzes: Hier geht der EuGH vom Leitbild des „mündigen“ Verbrauchers aus, nach dem z.B. Bedürfnissen des Gesundheits- oder des Verbraucherschutzes statt durch Vermarktungsverbote durch Vorschriften über die Etikettierung der Erzeugnisse Rechnung getragen werden könne. ${ }^{14}$ Aber auch im Bereich des Gesundheitsschutzes stellt der Gerichtshof (zumindest implizit) in manchen Urteilen die gewählte mitgliedstaatliche Konzeption bzw. das Schutzniveau in Frage. ${ }^{15}$

\section{Gefährdung des Schutzguts}

13 S. z.B. EuGH, Rs. C-293/94 (Brandsma), Slg. 1996, I-3159, Rn. 11 ff.; EuGH, Rs. C-67/97 (Bluhme), Slg. 1998, I-8033; EuGH, Rs. C-443/02 (Schreiber), Slg. 2004, I-7275; EuGH, Rs. C-434/04 (Ahokainen und Leppik), Slg. 2006, I-9171; EuGH, Rs. C-333/08 (Kommission/Frankreich), Slg. 2010, I-757, Rn. 85; EuGH, verb. Rs. C-338/04, C-359/04, C-460/04 (Placanica), Slg. 2007, I-1891; EuGH, verb. Rs. C-72/10, C-77/10 (Costa und Cifone), Urt. v. 16.2.2012; EuGH, verb. Rs. C-660/11, C-8/12 (Biasci u.a.), Urt. v. 12.9.2013; EuGH, verb. Rs. C-186/11, C-209/11 (Stanleybet International), Urt. v. 24.1.2013.

14 EuGH, Rs. 120/78 (Rewe), Slg. 1979, 649, Rn. 13 (Cassis de Dijon); EuGH, Rs. 178/84 (Kommission/Deutschland), Slg. 1987, 1227, Rn. 35; EuGH, Rs. 407/85 (Drei Glocken), Slg. 1988, 4233, Rn. 12 ff.; EuGH, Rs. C-14/00 (Kommission/Italien), Slg. 2003, I-513.

15 S. EuGH, Rs. C-108/09 (Ker-Optika), Slg. 2010, I-12213. 
Eine Rechtfertigung kann von vornherein nur unter der Voraussetzung in Betracht kommen dass tatsächlich nachvollziehbar eine Gefährdung des betreffenden Rechtsguts dargelegt wird, was ggf. eine (wissenschaftlich fundierte) Risikoanalyse impliziert. Allerdings ist den Mitgliedstaaten bei der hier erforderlichen Beurteilung ein gewisser Spielraum einzuräumen; zu berücksichtigen sind dabei der Wahrscheinlichkeitsgrad der Gefährdung sowie die potenzielle Schwere der Beeinträchtigung des Rechtsguts. ${ }^{16}$

\section{Geeignetheit und Erforderlichkeit}

Insbesondere die Erforderlichkeit hängt oft von der Einschätzung der Situation und einer notwendigerweise auch politisch geprägten Evaluation der in Betracht kommenden Instrumente ab. Das Unionsrecht soll den Mitgliedstaaten jedoch keine bestimmten politischen Optionen vorschreiben, so dass die Erforderlichkeit nur dann verneint werden sollte, wenn sich keine mildere Maßnahme aufdrängt. Im Übrigen genügt bei der Geeignetheit bereits ein Beitrag zur Zielerreichung. Insofern ist den Mitgliedstaaten bei der Beurteilung der Geeignetheit und der Erforderlichkeit bei tatbestandlichen Unsicherheiten ein gewisser Gestaltungsspielraum einzuräumen, wobei auch hier ggf. wissenschaftlich abgestützte plausible Nachweise zu erbringen sind.

So weist der EuGH denn auch häufig darauf hin, dass bei Zweifeln hinsichtlich der Wirksamkeit der Maßnahmen darauf abzustellen sei, dass (keine) Anhaltspunkte ersichtlich sein dürften, wonach die nationale Regelung das angestrebte Ziel nicht erreichen könne bzw. über das hinausgeht, was zur Zielerreichung notwendig ist, wobei ggf. eine Risikoanalyse und eine Alternativenprüfung vorzunehmen sind (so dass der Verhältnismäßigkeit auch eine gewisse verfahrensrechtliche Komponente zukommt). ${ }^{17}$

Grundsätzlich dürfte die Rechtsprechung denn auch dem hier zugrunde gelegten Konzept Rechnung tragen; der Umstand, dass einzelne Urteile mitunter nicht in jeder Beziehung überzeugen, dürfte konzeptionell in erster Linie darauf beruhen, dass der Gerichtshof hier die mitgliedstaatlichen Schutzkonzeptionen und -ziele in Frage stellt. ${ }^{18}$

\footnotetext{
16 S. z.B. EuGH, Rs. C-192/01 (Kommission/Dänemark) Slg. 2003, I-9693; EuGH, Rs. C-434/97 (Kommission/Frankreich), Slg. 2000, I-1129; EuGH, Rs. C-95/01 (Greenham), Slg. 2004, I-1333; EuGH, Rs. C-41/02 (Kommission/Niederlande), Slg. 2004, I-11375.

17 S. z.B. EuGH, Rs. C-473/98 (Toolex Alpha), Slg. 2000, I-5671, Rn. 40 ff.; EuGH, Rs. C-394/97 (Heinonen), Slg. 1999, I-3599, Rn. 36 ff.; EuGH, Rs. C-333/08 (Kommission/Frankreich), Slg. 2010, I757; EuGH, rs. C-219/07 (Nationale Raad van Dierekwekers), Slg. 2008, I-4475; EuGH, Rs. C-28/09 (Kommission/Österreich), Slg. 2011, I-13525.

18 So etwa in EuGH, Rs. C-108/09 (Ker-Optika), Slg. 2010, I-12213.
} 
Allerdings fällt insbesondere die Prüfung der Erforderlichkeit einer Maßnahme mitunter sehr engmaschig aus und dürfte nicht immer dem einzuräumenden Gestaltungsspielraum Rechnung tragen. ${ }^{19}$ Im Übrigen ist die Rechtsprechung insofern nicht immer ganz konsequent, als in gewissen anderen Urteilen die mitgliedstaatlichen Gestaltungsspielräume sehr (zu?) weit gefasst werden. ${ }^{20}$

\section{Schluss}

Insgesamt lassen sich somit bereits aus der Konzeption der Grundfreiheiten selbst einige Grundsätze in Bezug auf die Reichweite der mitgliedstaatlichen Gestaltungsspielräume ableiten. Die Rechtsprechung des EuGH trägt diesen prinzipiell auch durchaus Rechnung; allerdings sind nicht immer alle Aspekte deutlich formuliert, so dass hier eine etwas klarere Struktur - wobei in erster Linie die mitgliedstaatlichen Kompetenzen zur Definition der Schutzpolitiken von Bedeutung sind - wünschbar wäre. Sie könnte auch einen Beitrag zu einer größeren (dogmatischen) Kohärenz der Urteile leisten.

19 S. z.B. EuGH, Rs. C-28/09 (Kommission/Österreich), Slg. 2011, I-13525; EuGH, Rs. C-170/04 (Rosengren), Slg. 2007, I-4071.

20 S. insbesondere EuGH, Rs. C-388/95 (Belgien/Spanien), Slg. 2000, I-3123, Rn. 61 ff.; EuGH, Rs. C-108/01 (Prosciutto di Parma), Slg. 2003, I-5121; EuGH, Rs. C-469/00 (Ravi), Slg. 2003, I-5053; s. auch EuGH, Rs. C-220/98 (Lauder Cosmetics), Slg. 2000, I-117. 